\title{
Cultural Differences Compared with the Fusion of Chinese and Western Medicine Development
}

\author{
Yingmin Jia ${ }^{1 \#, a,}$, Li Yue ${ }^{2 \#, b}$ and Ruiyu $\mathrm{Li}^{3, \mathrm{c}, *}$ \\ ${ }^{1}$ PLA Army General Hospital,Beijing, 100700, China \\ 2Department of psychology, Chengde Medical College, Chengde, 067000, China \\ ${ }^{3}$ The Second Hospital of Xingtai Medical College, Xingtai, 054000, Hebei Province, China \\ cemail: Liruiyu651021@163.com \\ \#These authors contributed to this work equally. \\ ${ }^{*}$ Corresponding author: Ruiyu Li
}

Keywords: Cultural Differences; Comparison; Integration Development; The Combination of TCM and Western Medicine;

\begin{abstract}
In east and west two kinds of medicine, western medicine is a product of human wisdom and practice, the formation and development is inseparable from the specific cultural soil.The oriental culture and western culture in the process of form its own theoretical system without cross culture, thus formed the different cultural system and academic thoughts. Based on the comparison between east and west cultural roots, the philosophy of Chinese and western medicine, the way of thinking, research methods, and so on, Chinese and western medicine integration development, can be achieved on the new development level of the organic combination of Chinese and western medicine, should be combined with medical research direction in the future. The theory of complementary development of Chinese and western medicine is discussed.
\end{abstract}

\section{Introduction}

Differences between Chinese and Western medical theory and practice rooted in breeding and produce their cultural differences. What is culture? Culture is a social phenomenon, and is a member of a particular society and shared with each other to pass some kind of cultural identity and cultural collections, as an abstract concept in all aspects of social life. Broad definition is to all human creation called culture; narrow definition refers only to spiritual civilization, including things philosophy, religion, science, technology, literature, art, education, customs and other ideology. Chinese and Western medicine as the product of human wisdom and practical side of things, the formation and development is inseparable from the particular cultural soil, Eastern and Western cultures in the process of forming its own theoretical system are isolated from each other, resulting in a different cultural systems [1,2]. From the comparison of Eastern and Western cultural differences start with, and then discusses the integration of the two kinds of medicine.

\section{East-West Differences in Regional Cultural Environment Breeds Different Medical Systems}

Geographical environment and natural conditions of human existence and development of society and national substance essential prerequisite. Cultural differences were originally derived from differences in understanding of the natural world, the geographical conditions determine the initial direction of all nations and regional cultural development.

2.1 East Regional Environmental Culture and Traditional Chinese Medicine. Oriental culture originated in China Yellow River, the Yangtze River Basin. For thousands of years the Chinese people living here, creating a deep breadth of Chinese culture. Ancient Chinese side of the sea on three sides plateau relatively isolated geographical features, it provides the preconditions for the development of agricultural civilization. At the same time, self-sufficient economy continental rivers caused by the environment and its people firmly tied to the land. People seeking food instinct 
in search of food found in foods have healing effects and drugs, through labor to create a weapon, and produced Stone, bone needles and other medical appliances, early medical practice in this regard in the bud, and gradually formed a the theoretical system of medicine. Chinese culture and later spread to Japan, North Korea, South Korea, Southeast Asia, the formation of the so-called oriental cultural circle. This is only the history of human culture never ceased cultural system. Chinese medicine as an important part of Chinese culture, has become a never stopped medical system [3].

2.2 Regional Environment Western Culture and Western Culture. Western medicine originated in the Mediterranean ancient Greek culture, its marine environment in which the culture of Western peoples outside the original adventure pour national character. The ancient Greeks believed that human strength compared with the ocean is very small and fragile, but humans have to rely on itself courageous, resolute spirit conquered the sea, shaped the Western peoples open, courageous character. However, in the reign of religious darkness of medieval Europe, the West and cultural development stalled. Until the 15th century after the Renaissance to the 16th century, natural science, art has been an unprecedented development, and spread throughout Europe, spread to the Americas, Australia and other Western culture gradually formed, and the history of mankind to a new stage. Experimental and empirical approach based on medical research of "scholastic philosophy" a complete break, trained medical scientists of Western scientific awareness, so that Western medicine to get rid of the speculative reasoning mysticism and the achievements of biomedical science experiment mode [3].

\section{Philosophy East-west Cultural Differences Between Chinese and Western Medicine}

3.1 Philosophy of TCM. Oriental cultural influence of any one development of natural sciences will be subject to control and restrict philosophy. In the history of Chinese culture for thousands of years, resulting in different forms of philosophical thought, but the naive materialism and dialectics spontaneous thinking long-term is still dominated, the core idea is the strength theory, theory of yin and yang, the five elements theory, this pay attention to the overall macro, differentiation, interaction, unity of opposites, the internal contradictions of philosophy, and gradually formed the oriental cultural characteristics simple system theory thinking, this way of thinking in medicine and developed far-reaching. TCM theory philosophy under the guidance of "Heaven" philosophy, emphasizing the human body is an organic whole, people and the environment are intricately bound "natural" links, attention overall, Perpetual, with the natural organic view of nature. TCM clinical treatment focus from the whole process of change from the natural world to explore normal life activities and disease occurrence, development, evolution, attention to the function of organs organs and tissues as well as internal relations, to the harmony between man and nature. Philosophy in Medicine cover a wide range, can be roughly summarized as "Heaven" view of nature, "Form and Spirit," the whole concept of "syndrome differentiation" concept of treatment [4,5].

3.2 Western Medicine Importance Primitive Philosophy. Western philosophy from the atomistic mechanical materialism and elements of Ancient Greece, the Roman period, which is characterized as the composition of things, to inquire into the internal structure of the material. Its impact on Western medicine is mainly reflected in the dominance of primitive awareness, focusing on structural analysis, reducing research, analysis and macro-mechanical motion of individual forms of research thinking. Western mainstream philosophy that everything is primitive atom, microscopic particles, solid, combination, decomposability, external action as the core of reductionist thinking. Medical research and clinical practice in the Western philosophical thinking is formed Restoring Analysis of thinking, it is the basis of Western medical formation and development [5].

\section{East-west Cultural Differences Between Chinese and Western Medicine Way of Thinking}

People are exhibiting in the thinking process in a relatively stable form of brain activity called thinking, also known mode of thinking. Chinese medicine oriental cultural circle formed in the way of thinking, he thinks things are primitive vitality, "Yin" and "Yang" is the world of things are 
contradictory form of exercise, by the unity of opposites of yin and yang, each root interoperability, and mutual transformation to balance growth and decline reveal the essence of things and phenomena, which is based on the simple idea of system theory and methods of forming the core of the system, showing the image of thinking; thinking of Western medicine in Western cultural circles formed, its primitive understanding of things is atomistic, rather than on the strength, thereby causing it to mechanical theory of Natural Science as a guide. It considered contradictory form of exercise world by external force cause Relations "atom and void", the formation of atoms and the void is the essence of all things, it is reductionist thinking at its core ideas and methods of forming system, the performance of abstract thinking [5]. Eastern and Western cultures have a very clear difference in the way of thinking, its theoretical basis, clinical practice and research methods each have their own characteristics, combined with the deep study also difficult for the current Chinese and Western medicine.

\section{East-west Cultural Differences Among Western Medicine Research Methods}

Oriental culture in the "gas monism" philosophical point of view influences of ancient that gas is primitive substance constituting the universe, the movement is present in the form of gas and the inherent properties of the universe is all interlinked organic whole, the changes taking place in the universe and process, the result is air movement. Chinese medicine believes that life is a process of constant movement, the body is a form gas into motion and energy metabolic balance of the body, Chinese medicine theory has always stressed the use of dynamic image thinking, the use of a specific way of thinking analogy, deduction, outside hides, etc., understanding human health, long, strong, old, has been and analyze all pathological changes in the human body occurs after the disease, thereby grasp or predict development context disease, evolution trends, establish the appropriate therapeutic principle, give full play to guide clinical diagnosis and treatment effect, in order to achieve the fundamental purpose of seeking this treatment. Chinese medicine is a humanistic philosophy permeate the medical science, with strong characteristics of social sciences, research methods focus on integrity, having a system of Thinking. The influence of Western culture on Western medicine is mainly reflected in the primitive dominant understanding. Atomism the Western academic thought in the development process leading concept, which based on experiments, scientific theory of mathematical language, and gradually the research point of view to invest in solid organ or entity other aspects of substance, with the characteristics of the natural sciences. Mechanical view of nature in Western culture occupy a dominant position, when the mechanics and physics with great success, and promote the generation of reductionism. Under the guidance of ideological reductionism, Western medical research increasingly to the fine direction of depth from the whole organ, cell, has been deeply reach the cell, sub-cellular and molecular, genetic level [3,6]. Research Methods in Medicine relative to Western medical research is mechanical, not organic; is a limitation, rather than as a whole.

\section{Cultural Differences Western Disease Diagnosis and Treatment Decision Concept}

6.1 Nature of Traditional Chinese Medicine Clinic. Concept in oriental culture background, one hand on the basis of strength, emphasizing the human and the objective environment is unified, indivisible, whereby medicine proposed corresponding heaven, Five and Six, six exogenous kinky theory. On the other hand, the body itself is holistic, indissoluble, from this holistic to understand human physiology, pathology, medicine made Viscera, blood and body fluids, meridian theory. Chinese medicine believes that the physiological activities of human blood and body fluids is closely related to the time factor, and with varying periodicity, regularity. In the development and treatment of the disease process, "Yellow Emperor" with "Dan Hui", "Security Day", "Xi added," "Night and even" to represent the development of the disease varying periodicity and timing of [6,7]. Chinese medicine through four diagnostic differentiation, established hair cut herbs, to adjust the balance of yin and yang, the body ultimately achieve "Yin Pingyang secret, the spirit of governance." 
6.2 Western Medicine Treatment Concept of Entity. Western medicine is the study of the etiology under the guidance of reductionism, that the human body is composed of organs, tissues, cells, molecules constituting the entity, the entity mainly refers to a long, wide and high three-dimensional structure of the entity. Physiological and pathological conditions of the human body, it must pass a physical examination, chemical examination, biopsy and other means to find an objective entity has spatial structure as evidence, such as mycoplasma, chlamydia, viruses, bacteria and the like, and then subjected to drugs, surgery, or other Physical and chemical or biological means to control or eliminate disease was diseased tissue and diseased tissue cells or promote cell repair. Most Western medical theory is the direct study of the functional activity of the body's spatial structure and pathological changes.

6.3 Chinese and Western Medicine in Clinical Practice Objects. Practice the object is to establish discipline and social division mark,different focus and small differences between Chinese and Western medicine is often the watershed of two systems. Clinical treatment by taking into account the geographical environment, social status, physical and emotional factors in patients with seasonal climate, through governance "patient" to achieve the purpose of medical treatment, that is, "Heaven," the overall concept; Western medicine " reductionism "concept and experimental analysis methods enable researchers to enter the cell, sub-cellular and molecular level, through microscopic to understand the disease, focusing on cure" disease. "With the medical model to the bio - psycho - social medical model of change, only know how to cure a "disease" and do not understand the rule "patient" doctor, not a real doctor.

6.4 Chinese and Western Medicine Clinical Treatment Difference. Individual and Group of Eastern and Western cultures, has led to differences in clinical studies. Although Chinese and Western medicine on the same subjects, but in the methodology, medical model does not overlap. Chinese medicine stresses the "people-oriented", focusing on natural attributes and social attributes ideas while on therapy to diagnosis and treatment of individual-based, using different methods, due to the stock reform, with the addition and subtraction cards. Western medicine since the dynamic life processes stationary point, ignoring the impact of natural factors, the functional human body structure and various structures by means of instruments of observation and in-depth study of careful and detailed system to improve the pace of research, but the overall dynamic action conditions the lack of research and the complexity of the human dimension. The diagnosis is the corresponding frameworks, and standardized treatment model, as opposed to Chinese medicine, "individual medicine" is concerned, Western medicine is "community medicine" Both treatment modalities have a certain degree of rationality [6,7].

\section{Differences of Method Determine the Complementary Strengths of Chinese and Western Medicine}

In the history of the development of Chinese medicine for thousands of years, the "black box theory" formed a medicine holistic, systematic features to adjust the overall function led services. However, due to academic medicine is still in its simplicity so that the phenomenon of life and qualitative understanding of the macro, micro and quantitative understanding of the methodology is difficult to avoid the existence of limitations and blindness. Western medicine to restore the theory as the basis, to solve local lesions led Service, the "disease" and "patient" separated, ignoring the local and global relationships. However, the overall movement of the local law of motion there are differences in the nature, the natural sciences on the road unknowns are many, the human body is there are many phenomena, difficult to use today's scientific knowledge fully elucidated, if continues to abstract thinking and reductionism Guidance research, will leave more and more problems.

In the Eastern and Western cultural background of Chinese and Western medicine there are differences in the nature of its methodology is flawed and inadequate, but each with distinct characteristics. Since both the subject is a human practice, research task is to recognize the nature of the human body and disease, Western medicine reductionist research approach emphasizes local, and system analysis method is prominent in medicine as a whole. Future of medicine both require a 
large sample, multi-center, evidence based medicine, but also requires a highly individualized treatment, focusing on personality characteristics. With the advent of the era of globalization, one gradually interweave the East-West cultural integration made possible advantages of two kinds of Chinese and Western medicine complement each other, integration and development, to achieve the combination of Chinese and Western medicine in the new level of development, will become the medicine of the future the direction of research.

\section{References}

[1] Pingze Yong, Chinese and Western culture and Western medicine, Medicine and Philosophy, vol.10, pp. 48-50, 1997

[2] Shina Zhu, Comparison of Chinese and Western cultural characteristics from traditional Chinese medicine, JOURNAL OF SHANDONG UNIVERSITY OF TCM, vol.30, pp. 267-269, 2006.

[3] Zhengxin Mei, Jianmin Chen and Yongjun fee, From Civilization Thinking Understanding Integrative confusion Research, Traditional Chinese Medicine, vol.6, pp. 39-40, 2007.

[4] En Lee, Development philosophy and medicine, Chin J Diffic and Compl Cas, vol.6, pp. 382-383, 2007.

[5] Jiaying Min, Wang Yaping and En Lee, Chinese and Western ways of thinking from Comparison future development of Chinese medicine, Modern Journal of Integrated Traditional Chinese and Western Medicine, vol.22, pp. 340-342, 2013.

[6] Yumin He, Difference Confusion and Choice-Comparative Study of Chinese and Western medicine, Shenyang: Shenyang Press, pp. 305-32, 1990.

[7] Cai Hui and Zhiqiang Chen, Yanjun Prof Li En's Academic Thinking on Integerated Traditional and Western Medicine, Beijing: Beijing Science and Technology Press, pp. 80-109, 2009. 